\title{
History of the SPIE Medical Imaging Digital Pathology Conference
}

\author{
Anant Madabhushi $\odot^{\text {a,b }}$ and Metin N. Gurcan ${ }^{\mathrm{c}, *}$ \\ ${ }^{a}$ Case Western Reserve University, Cleveland, Ohio, United States \\ ${ }^{\mathrm{b}}$ Louis Stokes Cleveland Veterans Administration Medical Center, Cleveland, \\ Ohio, United States \\ ${ }^{c}$ Wake Forest School of Medicine, Winston-Salem, North Carolina, United States
}

\begin{abstract}
The term "digital pathology" (DP) broadly refers to the process of digitizing glass whole-slide images (WSIs) using a digital whole-slide scanner as these WSIs are also used to render a diagnosis. Artificial intelligence (AI), on the other hand, is broadly defined as the application of machine-based algorithms to make a prediction, just like an intelligent human who has access to the necessary knowledge to make said prediction. Deep learning is a specific type of $\mathrm{AI}$ that has become very popular for the analysis and interpretation of DP images over the last decade because of the recent increase in computational power and advancements in wholeslide scanning. AI-enabled DP analysis of routine hematoxylin and eosin-stained tissues has shown increasing utility in characterizing complex tissue architecture to render disease diagnoses, prognoses, and predicting therapeutic response. A PubMed search for "digital pathology" yielded 310 hits in articles published in 2020; for those published in 2010, the same search yielded only 12 hits. The Digital Pathology Conference was initiated in 2011 by the authors (Drs. Madabhushi and Gurcan), two early pioneers in computational pathology, in anticipation of the expected explosion of research and clinical interest in this space. ( 2022 Society of PhotoOptical Instrumentation Engineers (SPIE) [DOI: 10.1117/1.JMI.9.S1.012203]
\end{abstract}

Keywords: Digital Pathology Conference; SPIE Medical Imaging; conference history.

Paper 21308SSVR received Nov. 22, 2021; accepted for publication Feb. 7, 2022; published online Feb. 18, 2022.

The Digital Pathology Conference (DPC) was the last conference to be added to the SPIE Medical Imaging Symposium. ${ }^{1}$ The conference started in the 2013 meeting in Lake Buena Vista, Florida. Encouraged by the success of a special session on digital pathology that was held in conjunction with the three other conferences at the SPIE Medical Imaging Symposium in 2012, the symposium chairs, Drs. Nico Karssemeijer and Joseph Reinhardt, approached us (Madabhushi and Gurcan) to initiate an independent DPC for the 2013 symposium. We have been organizing several meetings annually since 2007 on this topic in North America and Europe, usually held in conjunction with other conferences, and we thought that SPIE Medical Imaging would be a good home for our ongoing meetings. Thus, DPC became the ninth conference to be included within the SPIE Medical Imaging Symposium. When we reached out to the leading scientists, researchers in this area in academia, government, and industry, they enthusiastically supported this effort. We had 23 people on our first scientific program committee.

The inaugural meeting received a relatively large number of submissions (50) to be presented as poster or oral presentations. Each submission was rigorously evaluated for possible inclusion by at least two experienced reviewers from within the program committee. Following the review phase, a total of 36 submissions were accepted (representing an overall acceptance rate of $72 \%$ ) for inclusion in the conference as either an oral or poster presentation. Based on the reviewer scores assigned to the individual papers, the top 20 were included as oral talks, and an additional 16 were selected as poster presentations.

The first DPC took place on February 2013 and began with a keynote presentation by Dr. Akira Saito from NEC Corp., Japan entitled "Dawn of the digital diagnosis assisting system, can it open a new age for pathology?" The presentation was very enthusiastically received by a packed audience in attendance. The keynote session saw 182 registered participants in

*Address all correspondence to Metin N. Gurcan, mgurcan@wakehealth.edu 
attendance (third highest among all nine conferences at the symposium). The DPC over the two days was also heavily attended. Similar to the other conferences, DPC offered "Best Poster" awards as well as "Best Student Paper" awards for every year.

The second DPC took place February 2014 in San Diego, California. In the second year of the conference, we were curious to see if we could repeat the first year's success. The meeting began with the plenary session entitled "Path, Present, and Future," delivered by Dr. Richard Levenson from the University of California, Davis Medical Center. Dr. Levenson's keynote talk on advances in digital and molecular pathology drew in over 250 attendees from various conferences, including Digital Pathology; Image Processing; Computer-Aided Diagnosis; and Image Perception, Observer Performance, and Technology Assessment; and resulted in some very lively discussion following the talk. The rest of the conference was similarly very heavily subscribed.

In the second year, we organized the very first workshop of our conference called "What do pathologists see on a slide: Implications for Digital Pathology." Three board-certified pathologists from the United States: Dr. John Tomaszewski, University at Buffalo, Dr. Ulysses Balis, University of Michigan, Dr. Richard Levenson, University of California, Davis Medical Center, and an academic pathologist from the United Kingdom, Dr. Darren Treanor, University of Leeds, presented a Pathology 101 for the SPIE Medical Imaging audience. The participating pathologists projected the digitized images of slides pertaining to renal disease, breast cancer, and prostate cancer and conversed with the audience on features that they saw on the slide. Dr. Tomaszewski presented a comprehensive list of challenges in the digital pathology image analysis to the SPIE Medical Imaging community, while Dr. Balis showcased how algorithms such as spatially invariant vector quantization can be used for high-throughput annotation of digitized slides. Drs. Treanor and Levenson took questions from the audience on some of the major challenges and opportunities for routine use and adoption of digital pathology and computerized image analysis in histopathology.

We organized yet another workshop during the third DPC that took place on February 2015 in Orlando, Florida. The meeting began on the evening of the 24th with a workshop entitled "Power of pathology: predicting disease aggressiveness from tissue slides" with expert pathologists Drs. John Tomaszewski (University of Buffalo), Ulysses Balis (University of Michigan), and Hannah Gilmore (Case Western Reserve University). Unlike the 2014 workshop that was intended as a "Pathology 101" for the SPIE Medical Imaging audience, the 2015 workshop challenged our expert panel to diagnose the presence of the disease on the slide and predict the aggressiveness of the disease and risk. The workshop began with an excellent review of breast cancer pathology and histopathology slide preparation by Dr. Hannah Gilmore. Subsequently, each of our expert pathologists reviewed a pathology slide corresponding to a different patient diagnosed with estrogen receptor-positive breast cancer. Following a didactic presentation of pathologic features identified on the image, each pathologist was asked to call on the level of risk (low, intermediate, or high). The risk predictions made by the pathologists based on the pathologic attributes were then compared against those obtained by a 21-gene expression assay called Oncotype DX. In every case, the predictions by the pathologist concurred with that obtained from the Oncotype DX test, revealing the ability of the pathologists to predict disease risk based on analysis of routine hematoxylin and eosin pathology slides alone. The workshop ended with a very lively interactive question and answer session between the audience and our expert panel of pathologists.

Over the years, the topics of the presented papers covered such diverse topics as imaging and pathology conversion, processing and storage of microscopic images, performance (e.g., speed, accuracy), and human factors, color standardization, detection, segmentation, immunohistochemistry (IHC) analysis, personalized medicine, computer-aided diagnosis, and multimodal image registration with histopathology. From the early years of the DPC in 2013 to 2016 to the current date, there has been a clear shift in the topics being presented at the meeting. For instance, there has been a clear migration from handcrafted and feature-engineered approaches to more of a deep learning/machine learning driven focus. In addition, while in the initial years, the focus tended to be on disease detection and disease grading ${ }^{2}$ (primarily prostate cancer, breast cancer, and lymphomas), recent papers have focused a lot more on risk stratification and treatment response assessment. In addition, the meeting has evolved, moving from a primarily oncology-driven focus to nononcologic applications including kidney, tuberculosis, and cardiovascular applications. In the initial years of the conference, there was a focus on work 


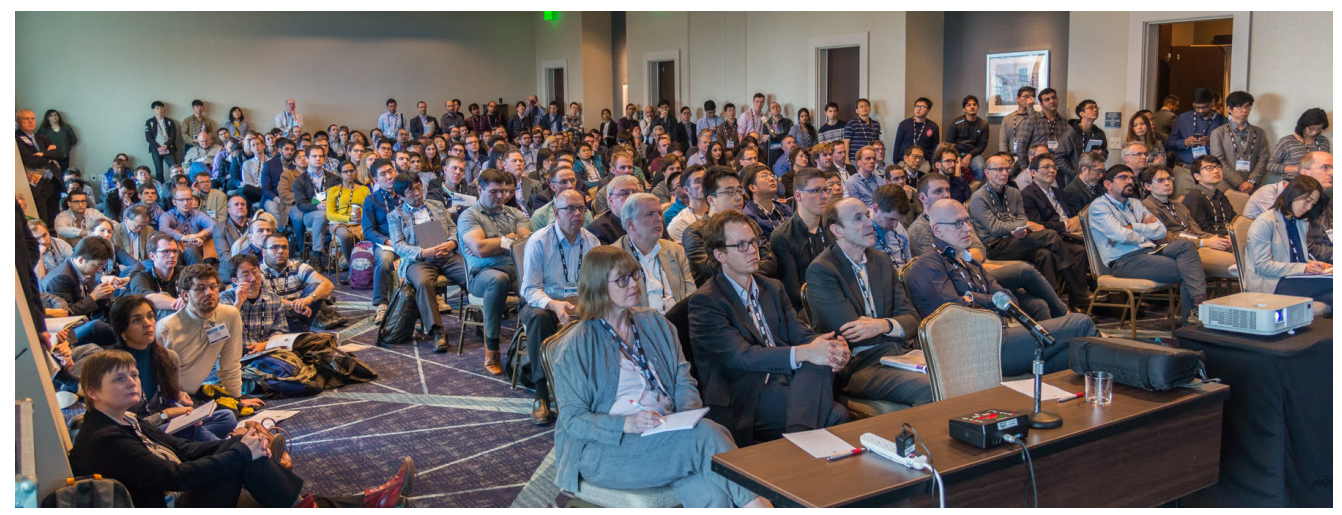

Fig. 1 The audience listening to Dr. Stumpe's Keynote talk at the 2018 DPC—part of the audience is another room because this room could not accommodate everybody. ${ }^{6}$

relating to visual perception, visual displays, and telepathology. However, over the years, the conference has begun to focus more squarely on AI and computational pathology, with deep learning-based approaches dominating.

Like the rest of the SPIE Medical Imaging Symposium, the DPC has been a student- and junior researcher-friendly conference. We experienced many—slightly nervous—students giving their first scientific talks and getting a warm welcome and support from the audience and session chairs.

The DPC featured several pathologists as attendees, workshop presenters, session chairs, program committee members, and presenters. It has been common to see several prominent radiologists in the SPIE Medical Imaging Symposium. ${ }^{3}$ Participating pathologists in our conference enriched the audience and content of the meeting. For example, the meeting in March 2016 in San Diego, California, formally began with a plenary talk given by Dr. Kenneth J. Bloom of Clarient Pathology Services entitled "Current challenges in digital pathology." $\mathrm{Dr}$. Bloom is a pathologist and an early adopter of information technology in pathology. Dr. Bloom discussed the current challenges in digital pathology from technical, regulatory, and clinical acceptance terms. The keynote talk drew in over 200 attendees from various conferences as part of the SPIE Medical Imaging Symposium.

The interest in the conference and keynote talks increased over the years. The sixth DPC took place in February 2018 in Houston, Texas, United States. The meeting formally began with a plenary talk given by Dr. Martin Stumpe, entitled "Advancing Cancer Diagnostics with Deep Learning." Dr. Stumpe, leading the pathology project at Google Research at the time, discussed algorithmic approaches for pathology, including deep learning and some of the challenges faced by researchers in the field. The keynote talk drew in 287 attendees from all the various conferences at the SPIE Medical Imaging Symposium. ${ }^{5}$ Because the largest meeting room was full, SPIE Administration decided to open a second room for the attendees to follow this presentation (Fig. 1).

One of the distinguishing characteristics of the SPIE meeting is that leadership always rotates, giving several distinguished members of the community a chance to serve our community. In 2017, Anant Madabhushi was replaced by John Tomaszewski, a practicing pathologist. In 2019, Metin Gurcan was replaced by Aaron Ward, a prominent image analysis researcher from Canada. The name of the conference was changed to Digital and Computational Pathology in 2020 to reflect better the intensive research representation of computational pathology in the conference. This year was also the year the conference was held virtually for the first time in its history because of Covid.

\section{Conclusion}

As mentioned in the introduction, the amount of interest in digital and computational pathology has exploded in the last decade. For instance, while two papers were published with the term computational pathology in 2014, a similar search on PubMed yielded 92 papers in 2021. While 
it would be hard to claim that this interest was directly a consequence of the initiation of the DPC, there is no doubt that the conference did provide some impetus for the increase in research interest in this space.

The continued success of the DPC is in no small part to the outstanding efforts of the program committee, who carefully constructed the program, provided valuable feedback to the authors, and chaired the sessions, SPIE Symposium Chairs, and SPIE staff, who kindly guided us through all the steps of the program organization and helped with all aspects of the program organization.

\section{Disclosures}

Dr. Gurcan: None. Dr. Madabhushi is an equity holder in Elucid Bioimaging and in Inspirata Inc. In addition, he has served as a scientific advisory board member for Inspirata Inc., Astrazeneca, Bristol Meyers-Squibb, and Merck. Currently, he serves on the advisory board of Aiforia Inc. and currently consults for Caris, Roche, Biohme, Castle Biosciences, and Aiforia. He also has sponsored research agreements with Philips, AstraZeneca, Boehringer-Ingelheim, Eli-Lilly, and Bristol Meyers-Squibb. His technology has been licensed to Elucid Bioimaging. He is also involved in three different R01 grants with Inspirata Inc.

\section{References}

1. M. N. Gurcan and A. Madabhushi, "Front matter: volume 8676," Proc. SPIE 8676, 867601 (2013).

2. K. Bera, I. Katz, and A. Madabhushi, "Reimagining T staging through artificial intelligence and machine learning image processing approaches in digital pathology," JCO Clin. Cancer Inf. 4, 1039-1050 (2020).

3. "SPIE Digital Library," 2022, https://www.spiedigitallibrary.org (accessed 11 February 2022).

4. M. N. Gurcan and A. Madabhushi, "Front matter: volume 9791," Proc. SPIE 9791, 979101 (2016).

5. J. Tomaszewski and M. N. Gurcan, "Front matter: volume 10581," Proc. SPIE 10581, 1058101 (2018).

6. "SPIE Medical Imaging 2018 photographs by Dr. Ken Hanson," 2018, https://photos.google .com/share/AF1QipNEeYHW3dGkt1iTUThHA9kMv4u7a07emJtyoRAxYFk8_b5fWUW_ 7Ajz7zGzxlUkWA/photo/AF1QipN-_5dXSZPSasill5E96F7SRQ81FZnGiX5EAE7A?key= UzdMQU43dFdONzB0RVdUYkpDX1pUT2FoR19oZ2NB (accessed 11 February 2022).

Dr. Anant Madabhushi is the Donnell Institute Professor in the Department of Biomedical Engineering at Case Western Reserve University. He is a research health scientist at the Louis Stokes, Cleveland, Virginia, USA. He has authored over 400 peer-reviewed publications and over 100 patents issued or pending. He is a fellow of the American Institute of Medical and Biological Engineering (AIMBE), and the Institute for Electrical and Electronic Engineers (IEEE), and the National Academy of Inventors (NAI).

Metin N. Gurcan, $\mathrm{PhD}$, is the founding director of the Center for Biomedical Informatics, professor of Internal Medicine and Biomedical Engineering at Wake Forest School of Medicine, and director of the Clinical Image Analysis Lab. He has authored over 200 publications and was awarded six patents. He received several awards, including the British FCO and NCI caBIG Embodying the Vision, NIH EUREKA. His informatics research has been supported by the National Institutes of Health (NCATS/NCI/NIDCD/NHLBINIBIB, and NIAID) and the Department of Defense. He is a fellow of SPIE. 